\title{
Dual source X-ray and electron SEM system: Elemental mapping of an Epithermal gold-bearing sample from Karangahake, New Zealand
}

Andrew Menzies and Stephan Boehm

Bruker Nano GmbH, Berlin, Berlin, Germany

Micro-X-ray fluorescence spectroscopy $[\mathrm{XRF}]$ is a long known and complementary analytical technique to e-beam Energy Dispersive Spectroscopy [EDS] for the characterization of the elemental composition within samples. The Bruker XTrace X-ray source enables the XRF technique to be utilized on a Scanning Electron Microscope [SEM]. Micro-XRF excitation analysis is also a small area (volume) technique ideal for low $\mathrm{kV}$ or beam sensitive samples as there are no charging effects (i.e., minimal sample preparation), the advantages of which include higher sensitivity for the detection of trace elements, higher energy Xray line excitation (full spectrum range up to $40 \mathrm{keV}$ ), as well as information from greater depth within the sample. The availability of modern X-ray polycapillary optics yields a X-rays focus spot size of less than $30 \mu \mathrm{m}$, all contained in an X-ray source that can be mounted on a SEM port. X-ray energy detection uses the existing Bruker EDS detector on the SEM. Accordingly, the SEM system will subsequently have dual source potential, i.e., both an electron and an X-ray source (Figure 1), which together offers new possibilities for material characterization.

The SEM user can operate a micro-XRF system using similar parameters as an e-beam system, yielding results comparable with traditional SEM-EDS analysis, whilst obtaining additional information from the X-ray source sample interaction. Electron excitation has a higher excitation efficiency for light elements (e.g., $\mathrm{Na}, \mathrm{Mg}, \mathrm{Al}, \mathrm{Si}$ ), and it is possible to perform measurements down to Boron. X-ray excitation efficiency is better for heavy elements (e.g., $\mathrm{Cr}, \mathrm{Mn}, \mathrm{Fe}, \mathrm{Co}$ ) and allows the detection of trace concentrations down to $10 \mathrm{ppm}$ for certain elements. The larger depth of X-ray excitation allows a deeper look inside the sample, allowing the characterization of relatively thick layers or even of multilayer systems starting from $1 \mathrm{~nm}$ and ranging to $40 \mu \mathrm{m}$, which is not possible with electron excitation. MicroXRF works with a fixed X-ray beam, consequently, X-ray elemental distribution maps must be acquired via stage movement. Developments of adding a piezo-based stage (Rapid Stage) mounted on top of the SEM stage enables high-speed elemental X-ray mapping over large areas (Figure 1), thus making it suitable for the analysis of larger samples (i.e., centimeter scale) that traditionally analysed in an SEM. Seamlessly integrated into Bruker ESPRIT software suite, it even allows for simultaneous electron and micro-XRF acquisition, incorporating light element spectral data as well as trace element and / or higher energy X-ray data. Sample preparation requirements are minimal for micro-XRF analysis and thus a wide range of sample types can be analyzed (e.g no carbon coating required; no high-quality polished surface required; low vacuum). Applications include economic geology, petrology, mining, mineral processing, forensics, environmental and archaeology, amongst others.

In this application relevant to the field of Geology, Exploration, Mining and Metallurgy, the objective is to determine elemental and mineralogical information about the sample to aid in understanding the geological processes and potential economic deposit genesis as well as mining, metallurgical and mineral processing considerations. This demonstrates the potential when utilizing the benefits of a combined electron and X-ray excitation system on a SEM. The selected sample is from a gold-bearing epithermal deposit (Karangahake) in New Zealand. Figure 1 is an image of the gold-bearing sample to be analyzed. The table in Figure 1 shows the relevant minerals and elements of interest. Figure 1 is a mosaic map of the elements of interest and the selected analytical conditions as measured using the micro-XRF source. In addition, in this sample it is possible to detect trace elements such as the K-Lines for Se, Ag, 
$\mathrm{Cd}$, and $\mathrm{Sb}$ that are not usually detectable by e-beam EDS as the concentrations are too low and/or the relevant element energy line is too high, and thus understand their distribution and mineralogical and textural relationships.

As can be seen in Figure 2, the micro-XRF hypermaps successfully identify the presence and location of gold $(\mathrm{Au})$ and silver $(\mathrm{Ag})$ bearing grains within the sample. In addition, it is possible to ascertain that these gold grains are primarily native gold or electrum. This large area map was achieved in 94 minutes. Furthermore, there are known peak overlaps for the various gold peaks, and thus an intensity map of a single Au element line might lead to misinterpretation. For example, the gold L- $\alpha$ line has an energy of $9.704 \mathrm{keV}$, which overlaps with $\mathrm{Zn} \mathrm{K}-\beta(9.569 \mathrm{keV})$ and W L- $\beta(9.682 \mathrm{keV})$, both of which occur in Au-bearing deposits. Thus, to confirm the presence of $\mathrm{Au}$, the information must be correct deconvoluted, and the presence of all the Au element lines should be confirmed. Figures 4 and 5 clearly demonstrate that gold is present in this sample using the functionality of the Maximum Pixel Spectrum, and interactively selecting individual pixels and groups of pixels within the hypermap.

Now that the presence of gold has been confirmed and the various positions of gold bearing grains identified, it is possible to analyse selected areas using the electron beam to yield higher resolution maps over specific areas. These follow-up analyses can be achieved by switching to e-beam SEM analyses (which requires sample coating and high vacuum) or selecting specific areas in the sample for further sample preparation prior to analysis. In this case the former is preferred, and examples of such maps are shown in Figure 6 and clearly highlight the fine scale relationship between Au and other elements associated with the mineralization such as $\mathrm{Zn}, \mathrm{Fe}, \mathrm{Cu}$, and $\mathrm{Pb}$. Thus, in the space of one analytical session, the benefits of dual source X-ray and electron SEM system in the analysis a gold-bearing sample were utilized. 

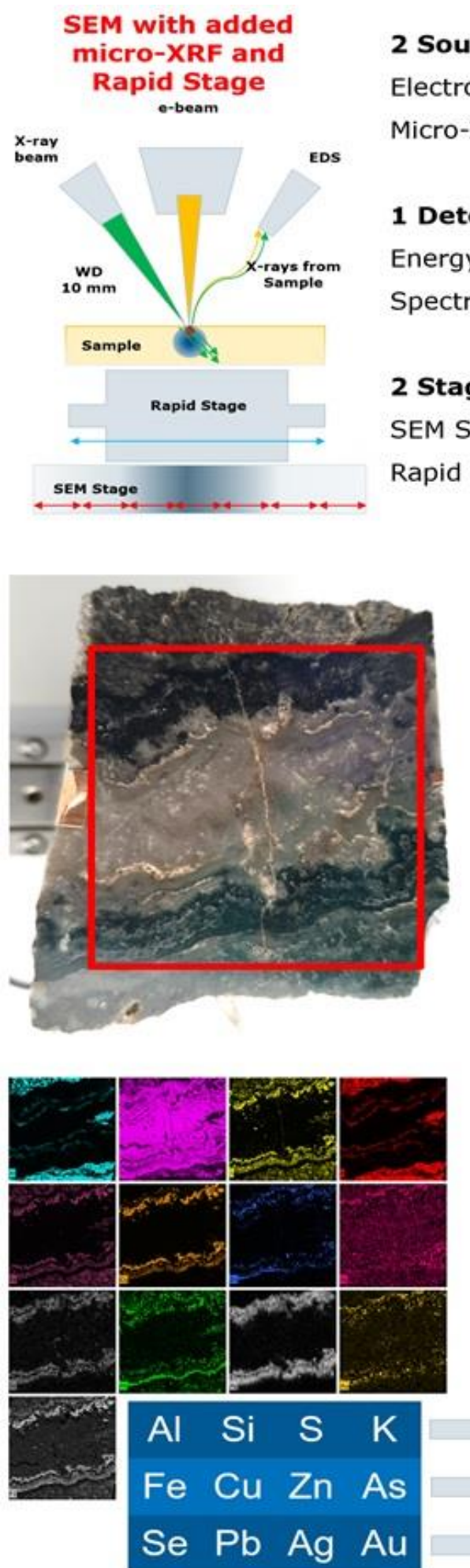

2 Sources:

Electron Beam (e-beam)

Micro-XRF (X-ray beam)

1 Detector:

Energy Dispersive

Spectrometer (EDS)

\section{Stages:}

SEM Stage

Rapid Stage
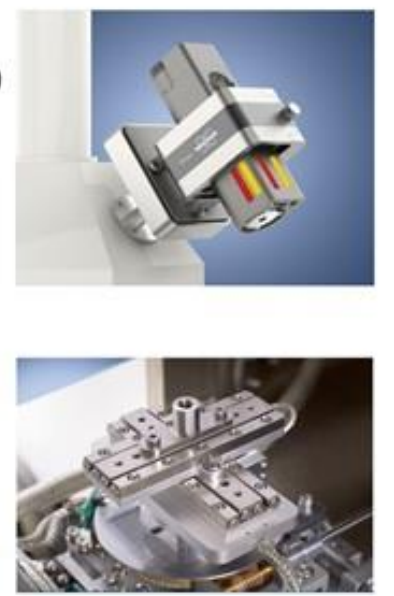

\begin{tabular}{|c|c}
\hline Mineral & Formula \\
\hline
\end{tabular}

\begin{tabular}{|l|l|}
\hline Native Gold & $\mathrm{Au}$ \\
\hline Native Silver & $\mathrm{Ag}$ \\
\hline
\end{tabular}

\section{Sulphides}

Pyrite $\mathrm{FeS}_{2}$

\begin{tabular}{l|l} 
Chalcopyrite & CuFeS $_{2}$ \\
\hline
\end{tabular}

\begin{tabular}{ll} 
Galena & $\mathrm{PbS}$ \\
\hline Sphalerite & $\mathrm{ZnS}$ \\
\hline
\end{tabular}

Gangue Mineralogy

\begin{tabular}{|c|c|}
\hline Quartz & $\mathrm{SiO}_{2}$ \\
\hline Adularia & $\mathrm{KAISi}_{3} \mathrm{O}_{8}$ \\
\hline
\end{tabular}

$\begin{array}{ll}\text { Beam: } & \text { X-ray } \\ \text { High Voltage: } & 50 \mathrm{kV} \\ \text { Anode Current: } & 600 \mu \mathrm{A} \\ \text { Analytical Spacing: } & \mathbf{1 0 0 ~ \mu \mathrm { m }} \\ \text { Dwell Time: } & \mathbf{3 2 0 0 0} \boldsymbol{\mu S} \text { (32 ms) } \\ \text { Analytical Area: } & 4.5 \times 4.5 \mathrm{~cm} \\ \text { Total Analytical Time: } & 94 \text { minutes } \\ & \\ \text { Spot Size: } & 25 \mu \mathrm{m} \\ \text { Interaction Depth: } & 10-100 \mu \mathrm{m}\end{array}$

Host Rock Elements: Al, Si, K

Mineralisation: $\mathrm{S}, \mathrm{Fe}, \mathrm{Cu}, \mathrm{Zn}, \mathrm{As}$

Economic Mineralistaion: Au, Ag, Se

Figure 1. Top Row: The micro-XRF works with a fixed beam and that cannot raster as a e-beam can. Consequently, a specialised stage for fast element distribution measurements with X-ray excitation is used. This stage can be mounted (temporary) on top of the existing SEM stage and easily removed. Middle Row: Gold-bearing Epithermal sample from Karangahake deposit, New Zealand. The Table shows the relevant minerals and elements of interest. The sample is carbon coated as both x-ray and electron analyse 
will be performed. Bottom Row: Large area X-ray map (45 mm x $45 \mathrm{~mm}$ ) of an Au-bearing Epithermal sample acquired with XTrace (Analytical Parameters: Tube Voltage: Rh at $50 \mathrm{kV}$; Anode Current: 600 $\mu \mathrm{A}$; Pixel Spacing: $100 \mu \mathrm{m}$; Analytical Time: 94 mins). Elemental Maps as indicated in Blue Box at bottom of image. Note the mineralisation, especially Ag and Ag along parallel veins.
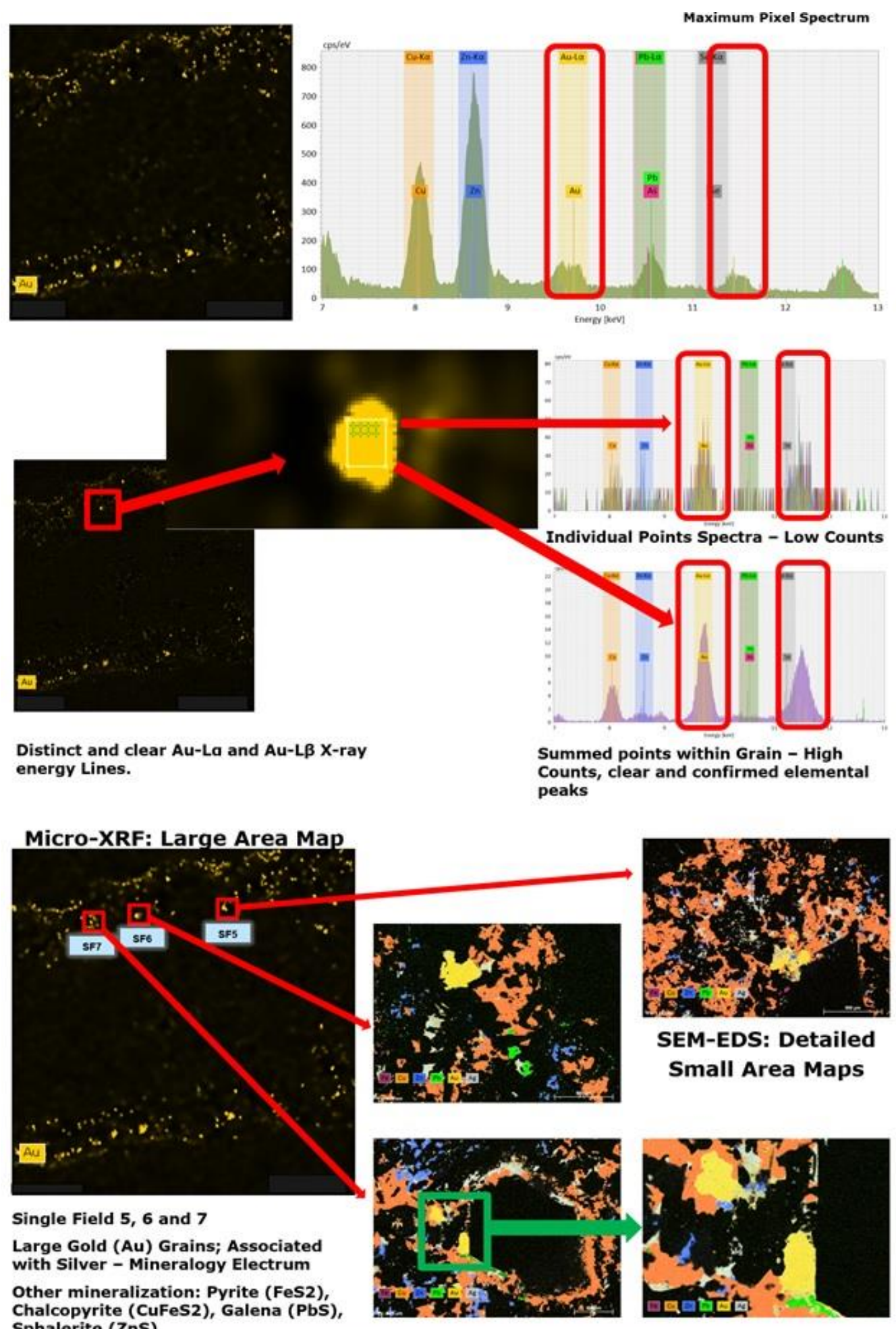
Figure 2. Top Row: Au Elemental Distribution. (Au-L $\alpha$ Energy Line $9.7 \mathrm{keV}$ ). Deconvolution Applied. Distinct and clear Au-L $\alpha$ and Au-L $\beta$ X-ray energy Lines. Presence of Au in the sample is confirmed. The Maximum Pixel Spectrum shows the maximum intensity measured for each energy channel for the entire map area. Middle Row: Au Elemental Distribution. (Au-L $\alpha$ Energy Line 9.7 $\mathrm{keV})$. Deconvolution Applied. Distinct and clear Au-L $\alpha$ and Au-L $\beta$ X-ray energy Lines. Presence of Au in the sample is confirmed. The Maximum Pixel Spectrum shows the maximum intensity measured for each energy channel for the entire map area. Bottom Row: Electron beam analysis of selected areas where gold grains are known to be present. 